Service social

\title{
L’accessibilité des services aux communautés culturelles : un principe ou une réalité?
}

\section{Nicole Boucher}

Volume 37, numéro 3, 1988

Index analytique 1978-1988

URI : https://id.erudit.org/iderudit/706414ar

DOI : https://doi.org/10.7202/706414ar

Aller au sommaire du numéro

Éditeur(s)

École de service social de l'Université Laval

ISSN

1708-1734 (numérique)

Découvrir la revue

Citer cet article

Boucher, N. (1988). L'accessibilité des services aux communautés culturelles :

un principe ou une réalité? Service social, 37(3), 455-462.

https://doi.org/10.7202/706414ar d'utilisation que vous pouvez consulter en ligne.

https://apropos.erudit.org/fr/usagers/politique-dutilisation/ 


\section{L'accessibilité des services aux communautés culturelles : un principe ou une réalité ?}

\section{Nicole Boucher}

Si, au Québec, I'accès aux services de santé et aux services sociaux est garanti par la Loi de la santé et des services sociaux, nous pouvons observer quelques lacunes importantes dans la mise en application de ce principe.

Depuis longtemps, les communautés culturelles du Québec signalent des difficultés concrètes d'accès aux services. Les obstacles majeurs identifiés par le comité sur l'accessibilité des services de santé et des services sociaux du réseau aux communautés culturelles ${ }^{1}$ sont le manque d'information, la faible présence des communautés culturelles dans le réseau, et surtout la persistance de barrières linguistiques et culturelles entre la clientèle et le personnel.

Suite à une description rapide des lacunes d'ordre législatif et administratif, je me propose de discuter ici diverses solutions envisagées.

\section{Une législation sociale exemplaire}

Le Québec dispose d'un ensemble de législations sociales parmi les plus progressistes au monde. La Loi de la santé et des services sociaux, à laquelle les Québécois sont très attachés, attribue au gouvernement et plus spécifiquement au ministre de la Santé et des Services sociaux la responsabilité de :

" rendre accessible à toute personne, d'une façon continue et pendant toute sa vie, la gamme complète des services de santé et des services sociaux, y compris la prévention et la réadaptation, de façon à répondre aux besoins des individus, des familles et des groupes aux plans physique, psychique et social ".

(article 3b) 
En plus d'assurer le droit aux services de façon universelle et d'encourager la participation à la gestion de ces services, la Loi de la santé et des services sociaux condamne explicitement toute forme de discrimination ou de racisme.

"Les services de santé et les services sociaux doivent être accordés sans distinction ou préférence fondée sur la race, la couleur, le sexe, la religion, la langue, l'ascendance nationale, l'origine sociale, les mœurs ou les convictions politiques de la personne qui les demande ou des membres de sa famille ».

(article 5)

La Charte des droits et libertés de la personne vient renforcer ces dispositions légales en protégeant l'accessibilité à l'emploi pour les groupes sociaux subissant une discrimination systémique sur le marché du travail, spécifiquement les femmes, les handicapés et les communautés culturelles.

Pourtant, bien que les droits aux services pour tous les citoyens du Québec soient explicitement établis et que le mandat du Ministère de la Santé et des Services sociaux soit clairement défini, les modalités concrètes d'application de ces droits entraînent indirectement l'exclusion de certains groupes sociaux tels que les communautés culturelles.

Conscient de ces lacunes, le gouvernement du Québec a promulgué, en décembre 1986, une déclaration sur les relations interethniques et inter-raciales dans laquelle il réaffirme sa volonté de veiller :

" à ce que soit respecté le droit de toute personne à l'égalité dans les domaines du travail, du logement, de la santé, des services sociaux, éducatifs ou des autres services offerts à la population, ainsi que dans l'accès aux lieux publics, sans discrimination fondée sur la race, la couleur, la religion, l'origine ethnique ou nationale ".

De plus, la Loi de la santé et des services sociaux a été modifiée en décembre 1986 par la loi 142, en précisant l'engagement du gouvernement québécois à ce que les services de santé et les services sociaux soient accessibles aux communautés culturelles dans leur "langue ». (article 3)

L'ensemble de ces dispositions législatives ne laissent aucun doute quant à la reconnaissance du droit des communautés culturelles aux services de santé et aux services sociaux. Pourtant, selon les représentants de divers groupes de pression " ethniques ", ces droits ne sont pas encore appliqués et restent souvent des vœux pieux. Serait-ce que la loi s'avère un instrument d'action sociale peu efficace? Pourquoi dépenser tant d'énergie à faire adopter des législations sociales si elles restent ensuite lettre morte? Est-ce que 
I'universalité des droits est une garantie suffisante pour les minorités?

\section{Des problèmes persistants}

Grâce à la politique canadienne encourageant l'immigration et la nationalisation, le Québec compte aujourd'hui plus de $10 \%$ de sa population désignée sous le terme vague de "communautés culturelles ". Ces Québécois « différents " se distinguent de la " majorité " par leur pays d'origine, leur origine ethnique, leur langue maternelle, leur race ou leur religion.

Juridiquement, leurs droits sont identiques à ceux de tous les citoyens canadiens et sont garantis par le gouvernement fédéral à travers sa politique d'immigration, la Charte canadienne des droits de la personne, les lois canadiennes d'accès à l'équité en matière d'emploi, la Constitution et la Déclaration universelle des droits de I'homme. Le Ministère de l'Immigration du Canada est, en principe, le responsable de l'accueil des immigrants et de leur intégration à la société canadienne.

Suite à l'entente fédérale-provinciale (entente Couture-Cullen) le Ministère des Communautés culturelles et de I'Immigration du Québec partage cette tâche de sélection, d'accueil, et assure la francisation et l'intégration des immigrants à la société québécoise.

$C^{\prime}$ est donc dire que plusieurs paliers de gouvernement (fédéralprovincial) et ministères ${ }^{2}$ sont concernés par le dossier de l'accessibilité des services de santé et des services sociaux aux communautés culturelles. Ce chevauchement de compétences entraîne des conflits entre ces diverses instances. Chacune rivalise dans la production de beaux discours à forte tendance électoraliste sans pour autant disposer de moyens concrets d'améliorer la situation.

Comment alors s'étonner de cette prolifération de législations et de déclarations de bonnes intentions de la part de nos gouvernements ? Comment s'étonner que le débat public autour de cette question reste essentiellement idéologique et politique, visant davantage la mobilisation de l'opinion publique par la dénonciation du racisme et de la discrimination que l'identification de mesures opérationnelles permettant d'améliorer la situation?

Car il ne faut pas s'y tromper, ce n'est pas la sensibilisation qui fait défaut mais plutôt une politique globale d'intégration économique et sociale qui dépasse le simple objectif de la francisation rapide des immigrants. 


\section{Des solutions problématiques}

Si le Ministère de la Santé et des Services sociaux a pour mandat d'assurer l'accessibilité des services à tous les Québécois y compris les membres des communautés culturelles, il assume cette tâche en tenant compte "de l'organisation et des ressources des établissements qui dispensent ces services » (article 4) et en répartissant entre ces services "les ressources humaines et financières de la façon la plus juste et rationnelle possible ». (article 3d)

Or, ces ressources sont loin d'être illimitées. Le Ministère en assure la répartition en fonction de problèmes sociaux importants, d'objectifs prioritaires et de populations-cibles, délaissant, par la même occasion, les "cas d'exception ». Les besoins " particuliers" des communautés culturelles en matière de santé et de services sociaux (ex. : réfugiés ayant subi des tortures, conflits conjugaux de mariages mixtes, conflits parents-enfants, difficultés d'intégration, etc.) et les rares difficultés de communication rencontrées, ont longtemps été considérés par le Ministère de la Santé et des Services sociaux comme relevant du Ministère des Communautés culturelles et de l'Immigration, spécifiquement mandaté pour faciliter la francisation et l'intégration.

Au Ministère des Communautés culturelles et de l'Immigration, ne disposant d'aucun service approprié pour répondre à des besoins individuels ou collectifs en matière de santé et de services sociaux, on se contente d'orienter " les cas » vers les établissements concernés et de "sensibiliser " ces établissements aux "différences culturelles ".

Ces dérobades provoquent, au sein des établissements et du personnel, des mouvements de résistance face à ce qu'ils considèrent une ingérence dans leurs affaires internes ou un transfert de charges sur un budget déjà insuffisant. Ces résistances sont facilement interprétées par certains comme du racisme et de la discrimination.

En fait, ces efforts de sensibilisation engendrent fréquemment la méfiance envers "la différence visible » et, sous prétexte de respect de la différence, le développement d'attitudes stéréotypées face aux " autres». Ainsi piégés, les nouveaux arrivants vivent une frustration croissante. Impuissants, ils subissent ces guerres de clochers, ces conflits bureaucratiques, en plus d'écoper, comme l'ensemble des Québécois, des limitations des ressources sociales gouvernementales, de la crise de l'administration sociale publique ${ }^{3}$ et des conflits nationalistes.

Heureusement, suite aux propositions de la Commission d'enquête sur la santé et les services sociaux présidée par monsieur Jean Rochon, le Ministère de la Santé et des Services sociaux se trouve présentement dans une phase de réorientation et de restructuration 
qui favorise la prise en compte des nouveaux besoins sociaux et, éventuellement, le développement de nouveaux services.

Le Ministère de la Santé et des Services sociaux s'est doté en 1988 d'un Bureau de coordination des services aux communautés culturelles et il vient d'adopter, en mai dernier, des orientations et un plan d'action pour 1989-1991, visant spécifiquement I'accessibilité des services aux communautés culturelles. ${ }^{4}$

Si cette conjoncture semble favorable à une amélioration sensible de la situation, n'oublions pas que le Ministère doit à la fois rendre le réseau accessible et bien le gérer. Confronté à la rareté des ressources et aux exigences de rendement, les services fonctionnent par priorités et par programmes s'adressant à des populations-cibles, ce qui favorise indirectement la sélection de clientèles et l'orientation d'une partie croissante des demandes vers des ressources " alternatives» (communautaires, bénévoles, privées ou " ethniques»).

Comment envisager une réelle amélioration de l'accessibilité sans une nouvelle croissance du réseau ? L'accès des services aux communautés culturelles ne peut être assuré aux dépens de l'ensemble des Québécois et vice-versa. L'accessibilité et l'équité sont difficiles à gérer dans un contexte de rareté des ressources.

En outre, il semble difficile de dégager un consensus entre les instances politiques et administratives concernées et entre les diverses communautés culturelles sur le type de mesures concrètes permettant de réaliser efficacement cette accessibilité. Certains réclament la création de programmes et de services spécifiques alors que d'autres veulent l'autonomie de gestion des établissements. (ex. : C.S.S. italien, C.L.S.C. grec, C.H. chinois, C.A. haïtien, etc.)

Est-ce là le meilleur moyen de respecter et de reconnaître les membres des communautés culturelles comme des Québécois à part entière et de faciliter leur intégration harmonieuse à la société ? Multiplier les ressources " ethniques " dans le réseau et hors réseau, est-ce rendre accessible universellement et équitablement les services ? Et comment financer un ensemble de services s'adressant à une clientèle restreinte? Comment accorder aux uns ce que I'on refusera aux autres ? Les critères de seuil critique des demandes ou de "territoires qui comptent une population multi-ethnique importante » (Plan d'action, p. 13) défavorisent nécessairement les communautés dont la population est peu nombreuse ou peu organisée, ou encore, bien intégrée (dans le sens ici de dispersée).

Quoi qu'il en soit, la rareté des ressources financières et humaines disponibles permet difficilement d'envisager de telles solutions pour l'ensemble des communautés. La mise en place de filières séparées pour les Québécois francophones, pour les anglophones et, finalement, pour les " autres ", afin d'éviter les conflits en 
limitant les contacts, n'est guère davantage souhaitable. Comment faire alors pour s'assurer que chacun reçoive un service personnalisé, approprié, tenant compte des différences culturelles, religieuses ou raciales?

\section{Un plan d'action discutable}

Le Ministère semble avoir opté pour un réaménagement majeur des ressources existantes afin de les "adapter » aux communautés culturelles par des modifications aux niveaux des pratiques de gestion, de l'organisation des services et des pratiques professionnelles. Les intervenants devront à l'avenir être à l'écoute des différences, être capables d'accepter cette différence et en mesure d'en tenir compte dans la distribution des services à cette clientèle (Plan d'action, p. 6). L'intégration de la dimension interculturelle dans tous les programmes du Ministère dépendra, en fait, de la "sensibilité " de chacun des membres du personnel aux réalités culturelles des clients et à leurs problèmes spécifiques.

Est-ce à dire que l'accessibilité n'est qu'une question de changement d'attitudes des Québécois vis-à-vis la différence ? La responsabilité de l'amélioration de l'accessibilité des services aux communautés culturelles repose-t-elle essentiellement sur les personnes ? Finalement, la question de l'accessibilité n'est-elle qu'un moyen "d'obliger » les Québécois à "s'adapter» aux exigences interculturelles d'une nouvelle société et d'accélérer l'embauche de "Québécois issus de diverses communautés ethno-culturelles minoritaires dans notre système socio-sanitaire et ceci à tous les niveaux »?

Cette vision bureaucratique d'un plan d'action est décevante autant pour les clients que pour les professionnels du réseau. Chacun reste confronté aux mêmes difficultés devant des situations de plus en plus complexes. L'accessibilité ne peut être assurée uniquement par des mesures bureaucratiques ou par des changements d'attitudes. Reconnaissons simplement que faute de moyens, l'accès direct pour tous, sans listes d'attentes, sans contraintes, sans frustrations, sans difficultés de communication d'ordre linguistique ou culturel, est de plus en plus illusoire.

Pourtant, l'adoption par le Ministère de mesures visant la transformation de l'ensemble du système socio-sanitaire pour satisfaire $20 \%$ de Québécois "dits différents " parce que considérés comme des victimes du système, même si ce fait n'a pu être formellement prouvé, démontre nettement la capacité des groupes minoritaires à utiliser le système à leur profit et, en conséquence, leur intégration réelle à la société québécoise. 
Politiquement rentable, la question de l'accessibilité restera un lieu de production de beaux discours grâce à la multiplication de "dossiers où la problématique est intégrée» ou " de plans régionaux de services " ou "de plans d'accès dans les établissements », ce qui n'assure en rien l'efficacité d'une intervention auprès d'une famille d'origine chinoise, ou haïtienne, ou italienne, ou libanaise, ou...

\section{Rendre accessible ou rendre efficace?}

Il faut reconnaître que l'intervention en contexte interculturel, du point de vue du professionnel, est une situation inconfortable, impliquant fréquemment conflits de cultures et de valeurs qui ne peuvent être résolus efficacement par décret ou par des mesures bureaucratiques. Faute d'habitudes, de modèles, de recettes applicables universellement à des situations diverses, faute de références communes, de préparation, de temps et de support, faute d'interprètes compétents, d'encadrement professionnel pertinent, faute de reconnaissance de leur compétence, les professionnels s'épuisent à tenter de répondre correctement aux " cas d'exception », tout en satisfaisant les exigences régulières de productivité.

Car il est difficile, pour tout intervenant, d'évaluer correctement la situation d'un jeune couple dont l'un des conjoints est d'origine vietnamienne par exemple, l'autre d'origine haïtienne, réclamant tous les deux la garde exclusive de l'enfant mâle nouveau-né. Ou encore, difficile d'identifier les préjugés, les perceptions négatives d'un client face aux travailleurs sociaux et de les corriger. Difficile de dépasser le malaise, le choc culturel subi de part et d'autre dans tout contact interculturel. Difficile d'éliminer le racisme chez les intervenants comme chez les clients. Difficile, voire impossible, de respecter des systèmes de valeurs ou des comportements contraires à la Loi de la protection de la jeunesse.

L'efficacité de l'intervention n'est-elle pas préférable à un accès formel aux services ? Elle dépend en grande partie du développement systématique de compétences nouvelles et d'habiletés à établir une relation de travail en contexte interculturel que seule une formation professionnelle approfondie permet d'acquérir.

\section{Notes}

\footnotetext{
${ }^{1}$ Rapport du comité sur l'accessibilité des services de santé et des services sociaux du réseau aux communautés culturelles, présenté par monsieur Christos Sirros, député de Laurier et adjoint parlementaire à madame Thérèse Lavoie-Roux, ministre de la Santé et des Services sociaux, Québec, novembre 1987.
} 
${ }^{2}$ Ministère de I'Immigration du Canada, Secrétariat d'État au multiculturalisme, Ministère des Communautés culturelles et de l'Immigration du Québec, Commission des droits de la personne.

${ }^{3}$ Voir Nicole Boucher, "Mieux administrer les services sociaux: une nécessité, un "défi ", revue Intervention, n 79, mars 1988.

${ }^{4}$ Bureau de coordination des services aux communautés culturelles, Accessibilité des services aux communautés culturelles, Orientations et Plan d'Action 1989-1991, Québec, Ministère de la Santé et des Services sociaux, mai 1989. 